\title{
Greyish-Black Rutile Megaclasts from the Nsanaragati Gem Placer, SW Cameroon: Geochemical Features and Genesis
}

\author{
Nguo Sylvestre Kanouo ${ }^{1,2}$, Rose Fouateu Yongue ${ }^{1}$, Shouyu Chen ${ }^{2}$, Emmanuel Njonfang $^{3}$, Changqian Ma ${ }^{2}$, Tanwi \\ Richard Ghogomu ${ }^{1}$, Jiangnan Zhao $^{2}$ \& Elise Sababa ${ }^{1}$ \\ ${ }^{1}$ Department of Earth Sciences, University of Yaoundé I, Yaoundé, Cameroon \\ ${ }^{2}$ State Key Laboratory of Geological Processes and Mineral Resources, China University of Geosciences, \\ Wuhan, China \\ ${ }^{3}$ Laboratoire de Géologie, Ecole Normale Supérieure, Université de Yaoundé I, Yaoundé, Cameroun \\ Correspondence: Nguo Sylvestre Kanouo, Laboratory of Applied Geology/Metallogeny, Department of Earth \\ Sciences, University of Yaoundé I, 812 Yaoundé, Cameroon. Tel: 237-7896-6240. E-mail: \\ sylvestrekanouo@yahoo.fr
}

Received: February 6, 2012 Accepted: February 14, 2012 Online Published: May 28, 2012

doi:10.5539/jgg.v4n2p134 URL: http://dx.doi.org/10.5539/jgg.v4n2p134

\begin{abstract}
Greyish-black rutile megaclasts from the Nsanaragati gem placer in south western Cameroon display a wide range of lithophile and siderophile elements in LA-ICP-MS analyses. $\mathrm{TiO}_{2}$ abundances exceed 94 wt.\%, with $\mathrm{FeO}$ (up to $4.2 \mathrm{wt} . \%$ ), $\mathrm{SiO}_{2}$ (up to $1.5 \mathrm{wt} . \%$ ) and $\mathrm{Al}_{2} \mathrm{O}_{3}$ (up to $1.8 \mathrm{wt} \%$ ) forming noticeable contents. Minor and trace elements with significant to moderate values (ppm) include $\mathrm{Nb}$ (965-4814), V (729-1846), Cr (495-756), $\mathrm{Ta}$ (44-180), and $\mathrm{Zr}$ (43-210). $\mathrm{Nb}$ /Ta ratios range between 10.0-44.9 and place the Nsanaragati rutile grains within the Niobium rutile. The measured contents for other elements including total REE are $<150$ ppm, mostly falling below detection limits. $\mathrm{Al}_{2} \mathrm{O}_{3}-\mathrm{MgO}$ plots (wt.\%) indicate that most rutile grains fall within a crust-derived rutile field, with rare plots in the mantle-derived field. $\mathrm{Cr}-\mathrm{Nb}$ plots suggest the grains are related to rutile from metapelitic rocks, rather than metamafic rocks. Temperatures calculated from $\mathrm{Zr}$ in rutile thermometry range from 470 to $675^{\circ} \mathrm{C}$, compatible with a likely crustal metapelitic source.
\end{abstract}

Keywords: Cameroon, greyish-black rutile, LA-ICP-MS, geochemistry, temperature

\section{Introduction}

Rutile is a common metamorphic rock forming mineral that crystallizes in wide range of temperature and pressure, significant for petrogenetic studies (Myron, 2003; Zack et al., 2004a; Xiong et al., 2005; Meinhold et al., 2008; Luvizotto \& Zack, 2009). This mineral crystallizes mostly in high grade metamorphic rocks: eclogites (Zack et al., 2002; Miller et al., 2007), some granulites and gneisses (Stendal et al., 2006; Meinhold, 2010); but can be found in some low grade metamorphic rocks: blueschist and greenschist (Meinhold, 2010). It is an accessory mineral in some magmatic rocks (Myron, 2003; Xiong et al., 2005; Dostal et al., 2009; Meinhold, 2010), and can crystallize in hydrothermal environments (Dostal et al., 2009; Garda et al., 2010). The high specific gravity $\left(4.2-5.6 \mathrm{~g} / \mathrm{cm}^{3}\right)$, hardness $(6.0-6.5)$ and resistance to chemical weathering of this mineral, facilitate its concentration in unconsolidated and lithified clastic sediments (Parfenoff et al., 1970, Zack et al., 2004b; Stendal et al., 2006; Dill et al., 2006, Birch et al., 2007; Rozendaal et al., 2009). Thus, rutile occurs in different geological environments and, consequently, complicates the study of displaced grains. When crystallizing, rutile incorporates chemical elements in minor and trace values (Zack et al., 2004a; 2004b; Luvizotto \& Zack, 2009), and other minerals: apatite (Miller et al., 2007); ilmenite or zircon (Meinhold, 2010), whose study helps rutile characterization and constrain its source parameters. It is mined for its high titanium contents and often contains minor and trace elements (Zack et al., 2004a; 2004b; Cherniak et al., 2007), important for correlative, petrogenetic and provenance studies (Triebold et al., 2007; Morton \& Chenery, 2009; Meinhold, 2010). Thus, rutile plays an important role in both economic and fundamental geology (Clack \& William-Jones, 2004; Klemme et al., 2005; Scott et al., 2005; Cerny et al., 2007; Dill et al., 2007; Meinhold, 2010). 
Mineralogical studies of the Nsanaragati gem corundum placer in the south western region of Cameroon, show rutile, zircon and ilmenite enrichment within the heavy minerals (Kanouo et al., 2012). No detailed study has been performed in the rutile grains. Their origin is still uncertain. Given the important role played by rutile, we present in this paper, the geochemical feature of greyish-black rutile megaclasts from this ore minerals deposition in other to contribute to its characterization and determine its origin.

\section{Geological Setting}

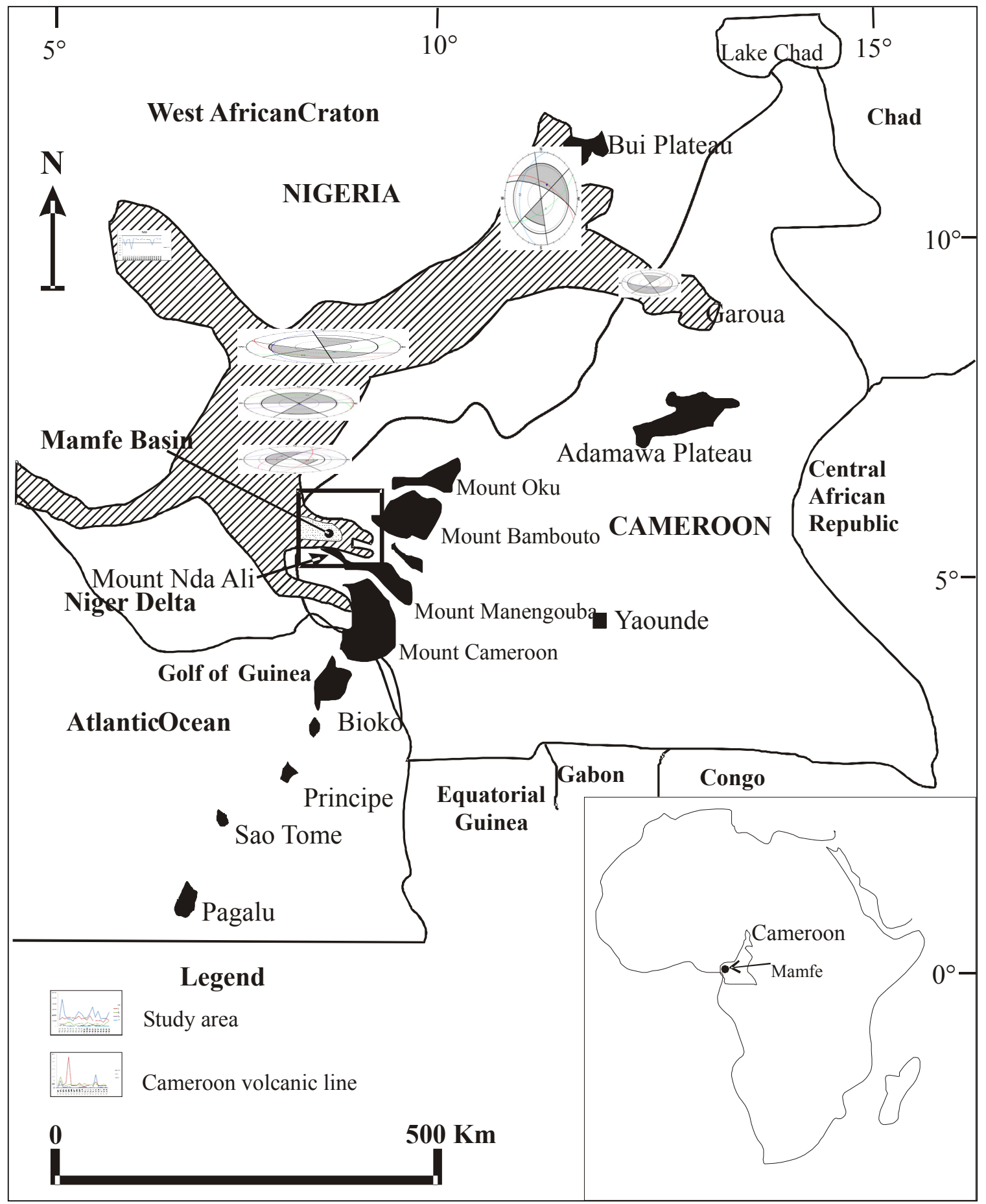

Figure 1. Location of the Mamfe sedimentary basin in the SW region of Cameroon and SE region of Nigeria by Benkhelil (1989) 
The Mamfe sedimentary basin in the south western region of Cameroon is one of the south eastern branches of the Benue Trough largely found in the Nigerian territory (Figure 1). The western part of this basin (Figure 2) is essentially filled by assumed Cretaceous age lithified clastic rocks of the Cross River Formation of Le Fur (1964-1965). These rocks are locally covered by the Nsanaragati and the Munaya gem placers (Laplaine \& Soba, 1967, Kanouo, 2008), and underlain by gneisses, schists, and granites of the Precambrian age basement (Wilson, 1928; Dumort, 1968). The sedimentary rocks in this area include immature sandstones and conglomerates, mudstones (Le Fur, 1964-1965; Eyong, 2003) of assumed Albian to Cenomanian age (Wilson, 1928; Le Fur, 1964-1965; Dumort, 1968). Recent works on the Nsanaragati gem-bearing revealed an important concentration of megaclastic rutile, sapphires and zircons within the heavy mineral suite (Kanouo, 2008; Kanouo et al., 2012). $\mathrm{U}-\mathrm{Pb}$ dating of reddish zircon grains provided an average age of $12.39 \pm 0.55$ Ma relating their sources to magmatic Serravalian age rocks (Kanouo et al., 2012).

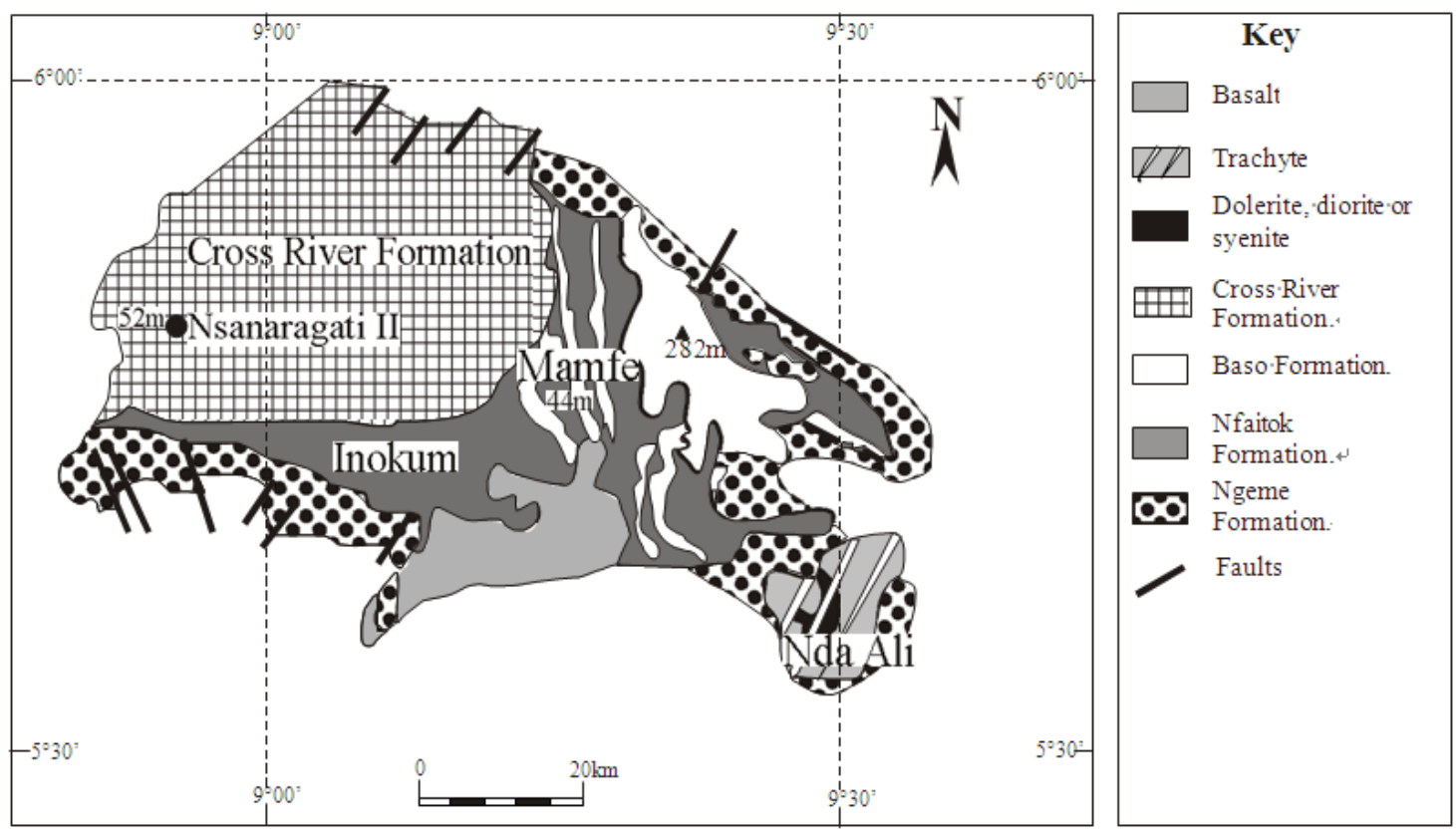

Figure 2. Geology map showing the distribution of lithology in the Mamfe sedimentary basin modified from Eyong (2003)

\section{Materials and Methods}

A total of nineteen greyish-black rutile megaclasts from the Nsanaragati gem placer deposit were morphologically examined at the laboratory of Applied Geology-Metallogeny, Department of Earth Sciences, University of Yaoundé I, Cameroon. They were characterized with a binocular microscope after separation of the coarse to very coarse grained opaque and non magnetic heavy minerals. The characterization was based on their physical properties including habit, shape, color and degree of roundness as presented by Duplaix (1958), Parfenoff et al. (1970), and Mange \& Maurer (1992).

The rutile megaclasts (NSG 1 - 19) were analyzed for their major, minor and trace element contents, using laser ablation inductively coupled plasma mass spectrometry (LA-ICP-MS) analytic techniques at the State Key Laboratory of Geological Processes and Mineral Resources at the China University of Geosciences in Wuhan (China). Each clast was mounted with epoxy resin on thin section and observed under a microscope directly linked to the Laser ablation system (LA). This system was used as the sampling tool for the Agilent 7500a quadrupole ICP-MS. The analytical protocol and data acquisition techniques are the same as in Liu et al. (2008) with GeoLas 2005 and Agilent 7500a ICP-MS instruments used for Laser sampling and acquisition ion-signal intensities. The carrier (He) and make-up (Ar) gas were mixed via a T-connector before entering the ICP. This mixture forms the central gas flow of the Ar plasma, in which $\mathrm{N}$ was added to decrease the detection limit and improve precision (Hu et al., 2008). Individual analysis was performed, using the Agilent Chemstation.

The thermometric calculation was done using the Zack et al. $(2004 \mathrm{~b})$ equations: $\mathrm{T}\left({ }^{\circ} \mathrm{C}\right)=127.8 * \ln (\mathrm{Zr} \mathrm{ppm})-10$. 


\section{Results}

\subsection{Rutile Morphology and Geochemistry}

The grain size ranges from 3 to $6 \mathrm{~mm}$. They are tabular, rounded or slightly elongated. The tabular grains are angular, whereas elongated and rounded grains are sub-blunt or blunt. The rutile megaclasts are all opaque with sub-metallic luster.

Over 45 chemical elements were analyzed in the Nsanaragati rutile megaclasts covering a wide range of lithophile and siderophile, with few chalcophile elements (Table 1). The concentration of these elements varies from one sample to another. $\mathrm{Ti}$ is the only quantified major element $\left(\mathrm{TiO}_{2}\right.$ content in Table 2, exceed $94.0 \mathrm{wt} . \%$ with a peak at $98.9 \mathrm{wt} \%$ (Figure 3). The other elements form the minor and trace elements suites.

Table 1. Qualitative classification of chemical element in the Nsanaragati greyish- black rutile megaclasts based on MacDonough and Sun (1995)

\begin{tabular}{ll}
\hline Lithophile elements & \\
Refractory & $\mathrm{Ti}, \mathrm{Be}, \mathrm{Zr}, \mathrm{Sc}, \mathrm{Ba}, \mathrm{Al}, \mathrm{V}, \mathrm{Nb}, \mathrm{Ta}, \mathrm{Hf}, \mathrm{Th}, \mathrm{U}, \mathrm{Y}, \mathrm{Sr}$, and REE (La Ce, Pr, Nd, Sm, Eu, \\
& $\mathrm{Gd}, \mathrm{Tb}, \mathrm{Dy}, \mathrm{Ho}, \mathrm{Er}, \mathrm{Tm}, \mathrm{Yb}, \mathrm{Lu})$ \\
Transitional & $\mathrm{Mg}, \mathrm{Si}, \mathrm{Cr}$ \\
Moderate volatile & $\mathrm{Li}, \mathrm{B}, \mathrm{Na}, \mathrm{K}, \mathrm{Rb}, \mathrm{Cs}$ \\
\hline Siderophile elements & \\
Refractory & $\mathrm{Mo}, \mathrm{W}$ \\
Transitional & $\mathrm{Fe}, \mathrm{Co}, \mathrm{Ni}$ \\
Moderate volatile & $\mathrm{P}, \mathrm{Cu}, \mathrm{Zn}, \mathrm{Ga}, \mathrm{Ge}, \mathrm{As}, \mathrm{Ag}, \mathrm{Sb}, \mathrm{Bi}$ \\
\hline Chalcophile elements & $\mathrm{Sn}, \mathrm{Pb}, \mathrm{Cd}$ \\
\hline Atmosphile elements & $\mathrm{O}$ “combined with other element” \\
\hline
\end{tabular}

Table 2. Major and minor elements abundance in the Nsanaragati greyish black rutile megaclasts

\begin{tabular}{|c|c|c|c|c|c|c|c|c|c|}
\hline sample & $\begin{array}{c}\mathrm{TiO}_{2} \\
(\mathrm{Wt} \%) \\
\end{array}$ & $\begin{array}{c}\mathrm{SiO}_{2} \\
(\mathrm{Wt} \%) \\
\end{array}$ & $\begin{array}{c}\mathrm{FeO} \\
(\mathrm{Wt} \%)\end{array}$ & $\begin{array}{c}\mathrm{Al}_{2} \mathrm{O}_{3} \\
(\mathrm{Wt} \%)\end{array}$ & $\begin{array}{c}\mathrm{Nb} \\
\left(\mathrm{ppm}^{2}\right) \\
\end{array}$ & $\begin{array}{c}\mathrm{V} \\
(\mathrm{ppm}) \\
\end{array}$ & $\mathrm{Nb} / \mathrm{Ta}$ & $\mathrm{Zr} / \mathrm{Hf}$ & $\mathrm{T}\left({ }^{\circ} \mathrm{C}\right)$ \\
\hline NSG01 & 98.7 & 0.39 & 0.31 & 0.067 & 1315 & 1160 & 16.75 & 49.76 & 673.37 \\
\hline NSG02 & 95.9 & 1.48 & 0.4 & 0.94 & 4814 & 1602 & 14.04 & 23.15 & 585.99 \\
\hline NSG03 & 98.5 & 0.4 & 0.38 & 0.072 & 1996 & 1332 & 11.08 & 21.71 & 655.78 \\
\hline NSG04 & 98.4 & 0.26 & 0.65 & 0.076 & 1335 & 1591 & 15.17 & 18.22 & 473.91 \\
\hline NSG05 & 94.4 & 0.47 & 4.16 & 0.2 & 1307 & 1579 & 15.04 & 28.72 & 620.63 \\
\hline NSG06 & 98.8 & 0.41 & 0.29 & 0.026 & 964 & 1527 & 21.56 & 14.10 & 470.68 \\
\hline NSG07 & 98.7 & 0.37 & 0.27 & 0.052 & 1803 & 1379 & 13.57 & 18.61 & 617.84 \\
\hline NSG08 & 98.4 & 0.48 & 0.29 & 0.02 & 2619 & 1846 & 44.85 & 19.09 & 573.59 \\
\hline NSG09 & 98.2 & 0.37 & 0.65 & 0.079 & 2222 & 1304 & 13.07 & 22.92 & 541.26 \\
\hline NSG10 & 98.6 & 0.40 & 0.26 & 0.065 & 1550 & 1445 & 11.05 & 20.63 & 571.58 \\
\hline NSG11 & 98.4 & 0.61 & 0.21 & 0.10 & 1498 & 1119 & 14.13 & 23.72 & 523.49 \\
\hline NSG12 & 98.5 & 0.29 & 0.36 & 0.041 & 2270 & 1911 & 12.67 & 25.56 & 522.3 \\
\hline NSG13 & 98.3 & 0.38 & 0.35 & 0.065 & 3454 & 1104 & 12.57 & 21.36 & 616.89 \\
\hline NSG14 & 98.5 & 0.53 & 0.32 & 0.054 & 1633 & 1073 & 10.57 & 20.64 & 560.6 \\
\hline NSG15 & 96.1 & 0.47 & 0.81 & 1.77 & 2687 & 978 & 13.06 & 21.91 & 537.09 \\
\hline NSG16 & 98.7 & 0.37 & 0.30 & 0.053 & 1326 & 1075 & 10.82 & 23.20 & 554.11 \\
\hline NSG17 & 98.8 & 0.33 & 0.31 & 0.080 & 1441 & 729 & 13.77 & 19.62 & 538.3 \\
\hline NSG18 & 98.5 & 0.51 & 0.29 & 0.058 & 1421 & 1416 & 14.62 & 20.61 & 507.37 \\
\hline NSG19 & 98.6 & 0.31 & 0.31 & 0.072 & 2511 & 1035 & 19.72 & 23.35 & 558.82 \\
\hline
\end{tabular}




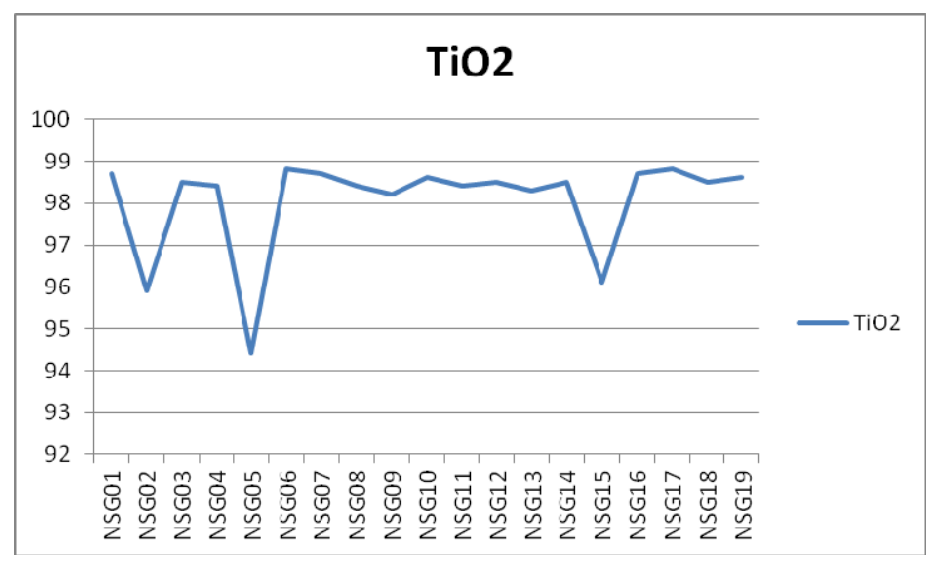

Figure 3. Plot diagram for $\mathrm{TiO}_{2}$ abundance in the Nsanaragati greyish rutile megaclasts

The minor element suites of the rutile grains (Table 2), plotted in Figures 4 and 5, include elements whose content range from 0.1 to 10 wt.\%. Some elements are expressed as oxide. $\mathrm{FeO}\left(0.20-4.20\right.$ wt.\%) and $\mathrm{SiO}_{2}$ (0.24- 1.48 wt.\%) contents are dominant while, $\mathrm{Nb}(964-4814 \mathrm{ppm})$ and $\mathrm{V}(729-1846 \mathrm{ppm})$ contents are significantly high. The highest $\mathrm{FeO}\left(4.20\right.$ wt.\%), $\mathrm{Al}_{2} \mathrm{O}_{3}\left(1.77\right.$ wt.\%) and $\mathrm{SiO}_{2}(1.48$ wt.\%) contents were respectively recorded in sample NGS5, NSG15 and NSG2, and distinguish these samples from the others.

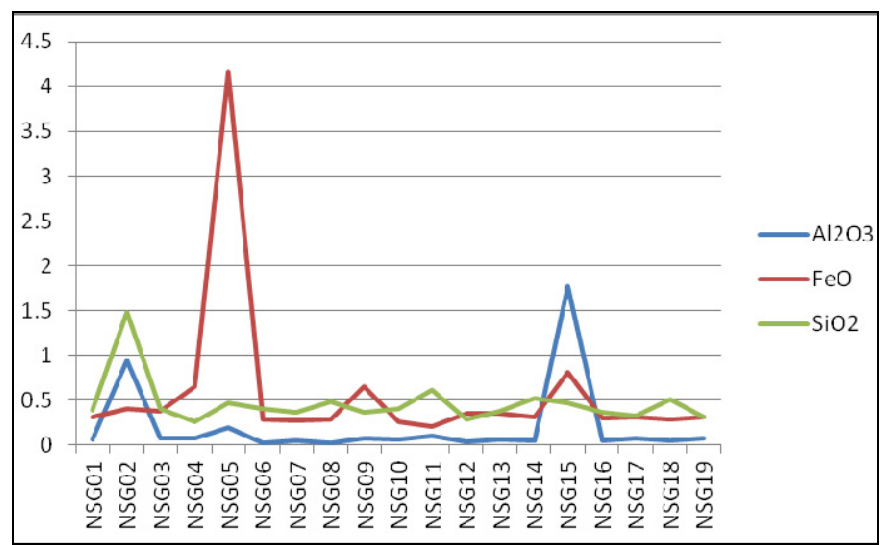

Figure 4. Plot diagram showing $\mathrm{Al}_{2} \mathrm{O}_{3}, \mathrm{FeO}$ and $\mathrm{SiO}_{2}$ variation within the Nsanaragati greyish-black rutile megaclasts

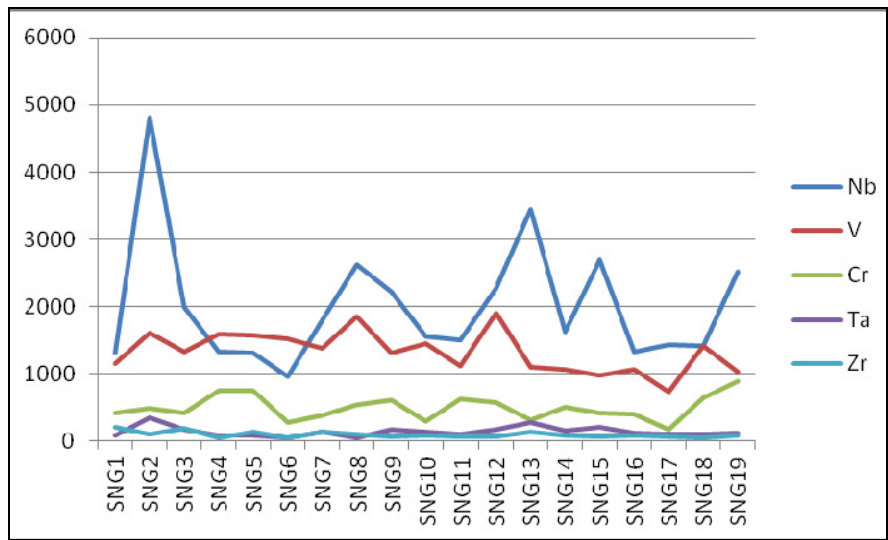

Figure 5. Plot diagram showing $\mathrm{Nb}, \mathrm{V}, \mathrm{T}, \mathrm{Cr}, \mathrm{Ta}$, and $\mathrm{Zr}$ variation within the Nsanaragati greyish-black rutile megaclasts 
The trace element suites in Table 3 and 4, include elements whose abundance is below $1000 \mathrm{ppm}$. With more than 37 elements in each rutile, trace elements are highly represented. Within these suites, $\mathrm{Na}, \mathrm{Mg}, \mathrm{P}, \mathrm{Ca}, \mathrm{Mn}$, $\mathrm{Zn}, \mathrm{K}, \mathrm{Cr}, \mathrm{W}, \mathrm{Zr}$, Ta, and Sn contents do not exceed 700 ppm, while, Pb, Hf, Ba, Sb, Mo, Sr, Cu, Ga, Co, U, Th, and $\mathrm{Sc}$ contents are below $50 \mathrm{ppm}$. The $\mathrm{Li}, \mathrm{Be}, \mathrm{B}, \mathrm{Co}, \mathrm{Cd}, \mathrm{Ni}, \mathrm{Ag}, \mathrm{Ge}, \mathrm{Rb}, \mathrm{Bi}, \mathrm{Cs}$, and $\mathrm{Y}$ contents are less than $10 \mathrm{ppm}$.

Table 3. Trace elements abundance in the Nsanaragati greyish- black rutile megaclasts

\begin{tabular}{|c|c|c|c|c|c|c|c|c|c|c|c|c|c|c|}
\hline sample & $\begin{array}{l}\mathrm{MgO} \\
(\mathrm{Wt} \%)\end{array}$ & $\begin{array}{l}\mathrm{P}_{2} \mathrm{O}_{5} \\
(\mathrm{Wt} \%)\end{array}$ & $\begin{array}{l}\mathrm{Na}_{2} \mathrm{O} \\
(\mathrm{Wt} \%)\end{array}$ & $\begin{array}{l}\mathrm{K}_{2} \mathrm{O} \\
(\mathrm{Wt} \%\end{array}$ & & & $\begin{array}{l}\mathrm{MnO} \\
(\mathrm{Wt} \%)\end{array}$ & $\begin{array}{l}\mathrm{Cr} \\
\text { (ppr }\end{array}$ & & $\begin{array}{l}\mathrm{Zr} \\
\text { (ppm) }\end{array}$ & $\begin{array}{l}\text { Ta } \\
(\mathrm{ppm})\end{array}$ & $\begin{array}{l}\text { Sn } \\
(\mathrm{ppm})\end{array}$ & $\begin{array}{l}\mathrm{Pb} \\
(\mathrm{ppm})\end{array}$ & $\begin{array}{l}\mathrm{U} \\
(\mathrm{ppm})\end{array}$ \\
\hline NSG01 & 0.015 & 0.0068 & 0.0027 & 0.0029 & 0.0 & & 0.0001 & 421 & & 210 & 78.5 & 25.9 & 4.44 & 10.2 \\
\hline NSG02 & 0.019 & 0.057 & 0.0031 & 0.0037 & 0.0 & & 0.0065 & 494 & & 106 & 343 & 28.7 & 218 & 2.75 \\
\hline NSG03 & 0.014 & 0.0047 & 0.0072 & 0.0032 & & & 0.0003 & 422 & & 183 & 180 & 24.6 & 21.1 & 12.7 \\
\hline NSG04 & 0.018 & 0.0034 & 0.0064 & 0.0006 & & & 0.016 & 756 & & 44.1 & 88 & 26.7 & 1.27 & 14.5 \\
\hline NSG05 & 0.07 & 0.03 & 0.018 & 0.017 & & & 0.027 & 755 & & 139 & 86.9 & 37.4 & 127 & 20.7 \\
\hline NSG06 & 0.016 & 0.0018 & 0.0013 & & 0.0 & & 0.0001 & 276 & & 43 & 44.7 & 13.5 & 1.23 & 0.65 \\
\hline NSG07 & 0.015 & 0.0059 & 0.0019 & 0.0008 & & & 0.0001 & 385 & & 136 & 133 & 34.6 & 2.55 & 20.1 \\
\hline NSG08 & 0.014 & 0.006 & 0.0014 & & & & 0.0002 & 534 & & 96.2 & 58.4 & 42.5 & 4.09 & 1.02 \\
\hline NSG09 & 0.018 & 0.0089 & 0.0034 & 0.0021 & & & 0.0004 & 607 & & 74.7 & 170 & 25.6 & 2.89 & 11.5 \\
\hline NSG10 & 0.015 & 0.0017 & 0.0024 & 0.0009 & & & 0.0006 & 304 & & 94.7 & 140 & 26.9 & 0.34 & 6.99 \\
\hline NSG11 & 0.016 & 0.012 & 0.012 & 0.0061 & 0.0 & & 0.0006 & 627 & & 65.0 & 106 & 26.9 & 15.4 & 10.8 \\
\hline NSG12 & 0.016 & & 0.0023 & 0.0005 & & & & 578 & & 64.4 & 179 & 23.1 & 3.23 & 1.74 \\
\hline NSG13 & 0.016 & & 0.0038 & 0.0012 & 0.0 & & 0.0036 & 308 & & 135 & 275 & 41.2 & 13.1 & 21.2 \\
\hline NSG14 & 0.014 & & 0.0038 & 0.0015 & & & & 499 & & 86.9 & 155 & 20.3 & 4.89 & 0.57 \\
\hline NSG15 & 0.015 & 0.032 & 0.0043 & 0.0025 & 0.0 & & 0.023 & 418 & & 72.3 & 206 & 25.6 & 14.9 & 1.04 \\
\hline NSG16 & 0.016 & 0.0041 & 0.011 & 0.0072 & 0.0 & & 0.0006 & 393 & & 82.6 & 123 & 20.9 & 7.32 & 14.2 \\
\hline NSG17 & 0.016 & 0.0037 & 0.0030 & 0.0016 & & & & 181 & & 73.0 & 105 & 36.4 & 2.92 & 4.70 \\
\hline NSG18 & 0.017 & 0.017 & 0.0027 & 0.0018 & & & & 648 & & 57.3 & 97.2 & 10.7 & 2.83 & 1.35 \\
\hline NSG19 & 0.013 & & 0.0023 & 0.0005 & 0.0 & & & 887 & & 85.7 & 127 & 22.0 & 1.82 & 1.92 \\
\hline sample & $\begin{array}{l}\mathrm{Sb} \\
(\mathrm{ppm})\end{array}$ & $\begin{array}{l}\text { Th } \\
\text { (ppm) }\end{array}$ & $\begin{array}{l}\mathrm{Zn} \\
(\mathrm{ppm})\end{array}$ & $\begin{array}{l}\mathrm{Ba} \\
(\mathrm{ppm})\end{array}$ & $\begin{array}{l}\mathrm{Be} \\
(\mathrm{ppm})\end{array}$ & $\begin{array}{l}\mathrm{Sc} \\
(\mathrm{ppm})\end{array}$ & $\begin{array}{l}\mathrm{Ga} \\
(\mathrm{ppn}\end{array}$ & & $\begin{array}{l}\mathrm{Hf} \\
(\mathrm{ppm})\end{array}$ & & $\begin{array}{l}\mathrm{Sr} \\
(\mathrm{ppm})\end{array}$ & $\begin{array}{l}\text { Mo } \\
\text { (ppm) }\end{array}$ & $\begin{array}{l}\mathrm{Ni} \\
(\mathrm{ppm})\end{array}$ & $\begin{array}{l}\text { Co } \\
\text { (ppm) }\end{array}$ \\
\hline NSG01 & 1.73 & 0.17 & 15.5 & 2.32 & 0.79 & 8.54 & 2.37 & & 4.22 & & 0.45 & 2.12 & & \\
\hline NSG02 & 4.9 & 65.8 & 17 & 146 & 1.56 & 9.79 & 7.31 & & 4.58 & & 31.2 & 1.43 & & 0.51 \\
\hline NSG03 & 1.08 & 4.51 & 4.79 & 3.43 & & 6.13 & 2.53 & & 8.43 & & 0.38 & 10 & & 0.3 \\
\hline NSG04 & 7.24 & 0.17 & 6.58 & 0.8 & 0.057 & 5.65 & 0.61 & & 2.42 & & 0.32 & 0.63 & & 0.21 \\
\hline NSG05 & 4.25 & 23.6 & 23.2 & 48.7 & & 11.5 & 8.46 & & 4.84 & & 4.52 & 5.2 & & 6.84 \\
\hline NSG06 & 1.36 & 0.046 & 4.28 & 0.76 & 0.22 & 6.03 & 0.62 & & 3.05 & & 0.34 & 0.58 & & 0.061 \\
\hline NSG07 & 5.95 & 0.17 & 5.28 & 1.09 & 0.26 & 6.4 & 0.49 & & 7.31 & & 0.37 & 4.11 & 3.8 & \\
\hline NSG08 & 0.36 & 0.32 & 4.3 & 1.54 & & 4.6 & 0.79 & & 5.04 & & 0.39 & 1.87 & & 0.2 \\
\hline NSG09 & 4.03 & 0.1 & 46.6 & 1.36 & 0.7 & 13.7 & 2.37 & & 3.26 & & 0.44 & 6.61 & 1.75 & 0.83 \\
\hline NSG10 & 233 & 0.087 & 3.22 & 0.99 & & 2.07 & 1.39 & & 4.59 & & 0.47 & 1.75 & & \\
\hline NSG11 & 18.6 & 1.82 & 7.10 & 12.0 & 0.48 & 10.5 & 1.87 & & 2.74 & & 0.92 & 0.87 & & 0.15 \\
\hline NSG12 & 162 & 1.48 & 5.14 & 0.64 & 0.46 & 3.88 & 0.76 & & 2.52 & & 0.25 & & 0.59 & 0.058 \\
\hline NSG13 & 7.69 & 3.76 & 23.5 & 4.82 & 0.29 & 25.3 & 1.97 & & 6.32 & & 0.49 & 11.9 & & 0.39 \\
\hline NSG14 & 242 & 0.17 & 7.60 & 1.74 & 0.45 & 3.14 & 0.66 & & 4.21 & & 1.00 & 0.86 & 2.16 & \\
\hline NSG15 & 237 & 2.46 & 9.79 & 4.70 & 72.5 & 4.99 & 4.44 & & 3.30 & & 0.65 & 2.63 & & 0.77 \\
\hline NSG16 & 50.5 & 0.63 & 9.16 & 3.89 & 0.11 & 6.62 & 2.28 & & 3.56 & & 0.61 & 1.42 & & \\
\hline NSG17 & 69.7 & 1.30 & 5.47 & 0.64 & 0.18 & 6.07 & 1.50 & & 3.72 & & 0.38 & 1.86 & & \\
\hline NSG18 & 113 & 0.14 & 4.89 & 0.38 & 0.34 & 10.9 & 0.65 & & 2.78 & & 0.24 & 0.69 & & 0.026 \\
\hline NSG19 & 120 & 1.53 & 4.93 & 0.98 & 0.17 & 2.94 & 1.82 & & 3.67 & & 0.39 & 0.15 & & 0.18 \\
\hline
\end{tabular}




\begin{tabular}{|c|c|c|c|c|c|c|c|c|c|c|}
\hline sample & $\begin{array}{l}\mathrm{Cu} \\
(\mathrm{ppm})\end{array}$ & $\begin{array}{l}\mathrm{Li} \\
(\mathrm{ppm})\end{array}$ & $\begin{array}{l}\text { B } \\
(\mathrm{ppm})\end{array}$ & $\begin{array}{l}\mathrm{Rb} \\
(\mathrm{ppm})\end{array}$ & $\begin{array}{l}\text { Y } \\
(\mathrm{ppm})\end{array}$ & $\begin{array}{l}\mathrm{Ag} \\
(\mathrm{ppm})\end{array}$ & $\begin{array}{l}\text { Cs } \\
(\mathrm{ppm})\end{array}$ & $\begin{array}{l}\mathrm{Bi} \\
(\mathrm{ppm})\end{array}$ & $\begin{array}{l}\mathrm{Cd} \\
(\mathrm{ppm})\end{array}$ & $\begin{array}{l}\mathrm{Ge} \\
(\mathrm{ppm})\end{array}$ \\
\hline NSG01 & 2.17 & & & 0.039 & 0.032 & 0.07 & 0.027 & 0.045 & & 0.097 \\
\hline NSG02 & 3.82 & 3.49 & 1.73 & 0.38 & 7.15 & 0.059 & 0.039 & 1.36 & & 1.79 \\
\hline NSG03 & 1.99 & 0.033 & 0.18 & 0.059 & 0.051 & & & 0.7 & & 0.78 \\
\hline NSG04 & 1.88 & 0.083 & 0.5 & 0.019 & 0.046 & 0.024 & 0.0036 & 0.0037 & & 1.08 \\
\hline NSG05 & 12.4 & 0.19 & & 0.16 & 0.17 & 0.083 & 0.013 & 1.53 & & 0.26 \\
\hline NSG06 & 1.8 & 0.037 & 0.29 & 0.15 & & & 0.021 & 0.018 & & 0.062 \\
\hline NSG07 & 2.06 & & & 0.019 & 0.016 & 0.084 & & 0.0074 & & 0.28 \\
\hline NSG08 & 1.52 & & 0.68 & 0.091 & 0.049 & & 0.0022 & 0.033 & & 0.23 \\
\hline NSG09 & 2.95 & 0.14 & 0.27 & 0.022 & 0.036 & & 0.016 & 0.051 & & 0.7 \\
\hline NSG10 & 1.78 & & 0.71 & & 0.26 & 0.15 & & 0.023 & & \\
\hline NSG11 & 2.38 & & & & 0.029 & 0.056 & 0.066 & 0.12 & & \\
\hline NSG12 & 0.92 & 0.0053 & 2.70 & 0.073 & 0.14 & 0.11 & 0.038 & 0.0026 & 0.22 & \\
\hline NSG13 & 3.03 & & bd & 0.037 & 0.064 & 0.036 & & 0.12 & & \\
\hline NSG14 & 2.83 & 0.084 & 0.70 & 0.066 & 0.045 & & & 0.015 & 2.74 & \\
\hline NSG15 & 2.59 & 0.029 & bd & 0.012 & 0.94 & 0.20 & 0.027 & 0.12 & & \\
\hline NSG16 & 2.76 & 0.055 & 0.57 & & & 0.089 & 0.021 & 0.027 & 1.66 & \\
\hline NSG17 & 2.34 & & 0.55 & & 0.28 & 0.014 & & 0.048 & & \\
\hline NSG18 & 2.26 & & 1.16 & 0.18 & & 0.032 & & 0.064 & & \\
\hline NSG19 & 2.54 & 0.032 & bd & 0.11 & 0.29 & 0.11 & & 0.055 & & \\
\hline
\end{tabular}

Table 4. Rare Earth elements abundances in the Nsanaragati greyish- black rutile megaclasts

\begin{tabular}{llllllllll}
\hline & $\begin{array}{l}\mathrm{La} \\
(\mathrm{ppm})\end{array}$ & $\begin{array}{l}\mathrm{Ce} \\
(\mathrm{ppm})\end{array}$ & $\begin{array}{l}\mathrm{Pr} \\
(\mathrm{ppm})\end{array}$ & $\begin{array}{l}\mathrm{Nd} \\
(\mathrm{ppm})\end{array}$ & $\begin{array}{l}\mathrm{Sm} \\
(\mathrm{ppm})\end{array}$ & $\begin{array}{l}\mathrm{Eu} \\
(\mathrm{ppm})\end{array}$ & $\begin{array}{l}\mathrm{Gd} \\
(\mathrm{ppm})\end{array}$ & $\begin{array}{l}\text { Tb } \\
(\mathrm{ppm})\end{array}$ & $\begin{array}{l}\text { Dy } \\
(\mathrm{ppm})\end{array}$ \\
\hline NSG01 & 0.14 & 0.54 & 0.022 & 0.23 & & 0.07 & & 0.0048 & 0.098 \\
NSG02 & 41.1 & 98 & 14.4 & 46.2 & 8.37 & 3.27 & 4.76 & 0.39 & 2.53 \\
NSG03 & 0.41 & 1.28 & 0.098 & 0.092 & 0.26 & & 0.19 & 0.025 & 0.04 \\
NSG04 & & 0.085 & & 0.18 & 0.068 & 0.0096 & & 0.0046 & \\
NSG05 & 5.08 & 7.92 & 0.88 & 2.78 & 0.083 & 0.082 & 0.72 & 0.023 & 0.046 \\
NSG06 & 0.025 & 0.11 & 0.031 & 0.25 & & & 0.2 & 0.018 & 0.069 \\
NSG07 & & 0.16 & 0.011 & 0.088 & 0.14 & 0.019 & 0.07 & & \\
NSG08 & 0.11 & 0.63 & 0.029 & 0.025 & & & 0.094 & 0.015 & 0.011 \\
NSG09 & 0.072 & 0.23 & & 0.097 & & & & 0.019 & 0.078 \\
NSG10 & 0.067 & 0.043 & & & 0.13 & 0.0091 & 0.032 & 0.0040 & 0.83 \\
NSG11 & 0.32 & 0.89 & 0.035 & & & 0.072 & & 0.032 & 0.086 \\
NSG12 & 0.23 & 0.32 & & 0.13 & & 0.035 & & 0.061 & \\
NSG13 & 0.64 & 1.04 & 0.068 & 0.39 & 0.072 & & 0.19 & & \\
NSG14 & 0.22 & 0.29 & 0.036 & 0.23 & & & & & 0.37 \\
NSG15 & 0.43 & 1.44 & 0.089 & 0.37 & & 0.11 & 0.30 & 0.021 & 0.16 \\
NSG16 & 0.36 & 0.38 & & 0.18 & 0.046 & & & & 0.28 \\
NSG17 & 0.17 & 0.24 & 0.13 & 0.21 & & 0.0080 & 0.19 & 0.039 & 0.091 \\
NSG18 & 0.022 & 0.33 & & 0.20 & & 0.034 & 0.24 & 0.023 & \\
NSG19 & 0.089 & 0.78 & 0.16 & 0.58 & 0.33 & 0.034 & 0.065 & & 0.088 \\
\hline & Ho & Er & Tm & Yb & Lu & TLREE & TMREE & THREE & TREE \\
$(\mathrm{prg})$ & $(\mathrm{ppm})$ & $(\mathrm{ppm})$ & $(\mathrm{ppm})$ & $(\mathrm{ppm})$ & $(\mathrm{ppm})$ & $(\mathrm{ppm})$ & $(\mathrm{ppm})$ & $(\mathrm{ppm})$ \\
\hline NSG01 & & 0.043 & & & 0.013 & 0.702 & 0.3 & 0.159 & 1.1608 \\
NSG02 & 0.37 & 0.75 & 0.15 & 0.73 & 0.087 & 153.5 & 62.6 & 5.007 & 221.107 \\
\hline
\end{tabular}




\begin{tabular}{llllllllll}
\hline NSG03 & 0.0047 & 0.044 & & 0.1 & 0.02 & 1.788 & 0.542 & 0.234 & 2.5637 \\
NSG04 & 0.0047 & 0.014 & & & 0.016 & 0.085 & 0.258 & 0.039 & 0.3819 \\
NSG05 & 0.034 & & & 0.14 & 0.01 & 13.88 & 3.665 & 0.253 & 17.798 \\
NSG06 & 0.0067 & 0.013 & 0.01 & 0.022 & 0.0044 & 0.166 & 0.45 & 0.143 & 0.759 \\
NSG07 & & 0.055 & 0.0076 & & & 0.171 & 0.317 & 0.063 & 0.551 \\
NSG08 & 0.01 & & 0.016 & & 0.011 & 0.769 & 0.119 & 0.063 & 0.951 \\
NSG09 & & & & & 0.0066 & 0.302 & 0.097 & 0.104 & 0.503 \\
NSG10 & & & 0.036 & & & 0.110 & 0.172 & 0.867 & 1.149 \\
NSG11 & 0.016 & & 0.0061 & & & 1.238 & 0.072 & 0.139 & 1.449 \\
NSG12 & & 0.14 & & 0.10 & & 0.544 & 0.167 & 0.301 & 1.011 \\
NSG13 & & 0.039 & & 0.24 & 0.025 & 1.743 & 0.655 & 0.306 & 2.703 \\
NSG14 & & 0.028 & 0.029 & 0.22 & & 0.539 & 0.233 & 0.641 & 1.414 \\
NSG15 & 0.016 & 0.17 & & & 0.054 & 1.956 & 0.777 & 0.419 & 3.153 \\
NSG16 & 0.029 & 0.15 & 0.013 & 0.13 & & 0.742 & 0.228 & 0.603 & 1.573 \\
NSG17 & & & 0.026 & & & 0.538 & 0.407 & 0.156 & 1.101 \\
NSG18 & 0.024 & 0.20 & & 0.14 & & 0.352 & 0.476 & 0.381 & 1.208 \\
NSG19 & 0.017 & 0.16 & 0.053 & 0.068 & & 1.038 & 1.011 & 0.381 & 2.424 \\
\hline
\end{tabular}

The rare earth element (REE) abundances (up to $98 \mathrm{ppm}$ ) in Table 4 are low falling within the range of trace elements. The relative low contents for rare earth elements and most of the trace elements show that they do not readily enter rutile structure during crystallization (Miller et al., 2007). The total REE (TREE) abundance ranges from 0.3 to $221.2 \mathrm{ppm}$, with the highest value been recorded in sample NSG2; differentiating this sample from the others.

\section{Discussions}

\subsection{Clastic Rutile Morphology}

The grain size of the Nsanaragati grayish rutile megaclasts is up to $6 \mathrm{~mm}$, and fall within the range of gravels or pebbles clasts in Wentworth (1922) clastic particles size classification table. The size of the rutile grains suggests that they were probably sorted from course to very coarse grained rocks. This interpretation is based on James (1851) and Roux and Rojax (2007) characterization of other clastic minerals. For these authors, the grain size of hard and weathering resistant displaced and deposited minerals such as rutile, quartz, tourmaline, kyanite, and zircon highly depends on the textural feature of their source rocks.

The studied rutile are mostly angular or blunt in feature and therefore show some morphological differences that help for the discrimination of their source parameters; as Cailleux (1964), Berthois (1970) and Selley (1982) for other clastic minerals. The angular nature is a conserved primary relic feature during transportation. These angular grains were probably sorted not too far from their source area, and may be product from local sedimentation (Cailleux, 1964; Berthois, 1975). As suggested by Kanouo et al. (2012), the blunt nature of the rutile grains may be due to constant reworking or long distance hydro-mechanical transportation in water from distal sources.

\subsection{Geochemical Characteristic and Correlation}

The Nsanaragati rutile megaclasts appear as an important reservoir of minor and trace elements (up to 45 elements were identified). The element contents vary from one sample to another. $\mathrm{TiO}_{2}$ content exceed $94 \mathrm{wt} \%$, and some values are similar to those of Garon Lake (Cu-Zn) ore (Clack \& William-Jones, 2004) and Yaoundé rutile (Stendal et al., 2005); although major correlations do not exist for other chemical elements.

The FeO values (0.20- 4.20 wt.\%) contents are significant. The FeO enrichment in sample NSG5 (> 4.0 wt.\%) differentiate this sample from the others. The highest $\mathrm{MgO}\left(0.07\right.$ wt.\%), $\mathrm{Na}_{2} \mathrm{O}\left(0.018\right.$ wt.\%), $\mathrm{K}_{2} \mathrm{O}(0.017$ wt.\%), and $\mathrm{MnO}\left(0.027\right.$ wt.\%), and lowest $\mathrm{TiO}_{2}(94.4 \mathrm{wt} . \%)$ contents were also measured in this sample. That shows a possible enrichment of $\mathrm{Fe}, \mathrm{K}, \mathrm{Na}$, and $\mathrm{Mn}$ in the source environment. Correlation between these elements may be suggested. This geochemical anomaly differentiates NSG5 from the others, and may relate their source to Fe enriched and Ti fairly depleted environment. In general, Fe contents of the studied rutile grains exceed $0.2 \mathrm{wt}$. \%, and such values have been attributed to the presence of ilmenite lamellar in inclusion (Meinhold, 2010). Another feature of NSG5, is the relative high content in LREE (13.88 ppm). It is the second highest values after that of 
sample NSG2 (153.5 ppm). This sample also has the highest $\mathrm{SiO}_{2}(1.48 \mathrm{wt} . \%)$ and $\mathrm{P}_{2} \mathrm{O}_{5}(0.03$ wt.\%). The high $\mathrm{P}_{2} \mathrm{O}_{5}$ and LREE content in this grain can be attributed to apatite inclusion. This has been reported in some gabbroic and eclogitic source rutile from eastern Alps (Miller et al., 2007). Sample NSG15 is rather characterized by the high $\mathrm{Al}_{2} \mathrm{O}_{3}$ contents $\left(1.77\right.$ wt.\%) with $\mathrm{P}_{2} \mathrm{O}_{5}$ and $\mathrm{SiO}_{2}$ similar to those of NSG5. That may relate its crystallization to an aluminum rich environment.

The $\mathrm{Cr}$ and $\mathrm{Nb}$ values in the rutile megaclasts range from 495 to $756 \mathrm{ppm}$, and, 964 to $4814 \mathrm{ppm}$ respectively, fall within the range values of Triebold et al. (2007) and Meinhold et al. (2008) metapelitic rutiles. For Meinhold et al. (2008) rutile from metapelitic source rock are characterized by $\mathrm{Cr}<\mathrm{Nb}$ and $\mathrm{Nb}>800 \mathrm{ppm}$ (eg micaschists, felsic granulites), while, those from metamafic rocks (eg eclogites and mafic granulites) have low $\mathrm{Nb}(\mathrm{Nb}<800$ $\mathrm{ppm}$ ). If based on this hypothesis, all the Nsanaragati rutile grains are within the range for metapelitic rutile. The plotted data in $\mathrm{Nb}$ versus $\mathrm{Cr}$ (Figure 6) confirm their relation with metapelitic source rutiles. They were probably crystallized in metapelitic rocks. Some of the studied rutile megaclasts reflect moderate $\mathrm{Cr}$ and $\mathrm{V}$ correlations that may indicate mafic igneous contribution for the crystallization of some the rutile (Rosendaal et al., 2009).

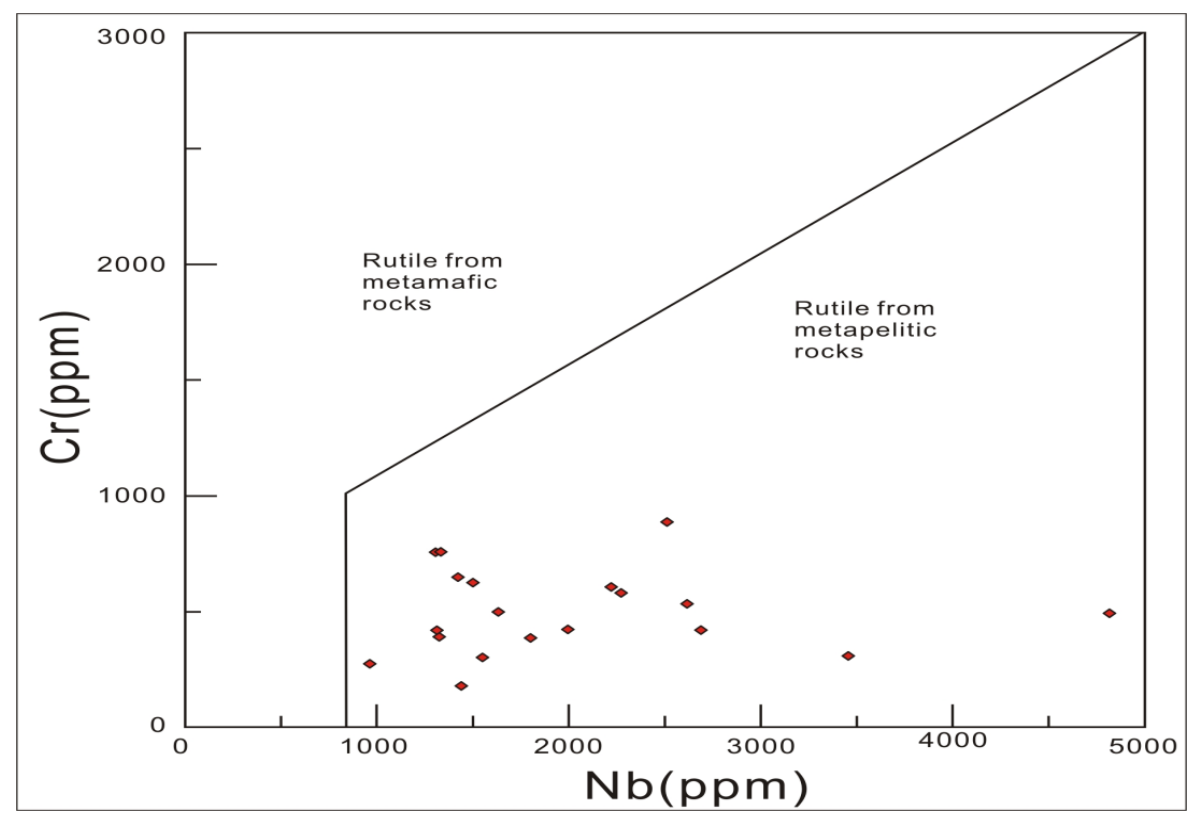

Figure 6. Nsanaragati rutile $\mathrm{Nb}$ and $\mathrm{Cr}$ abundance plotted in Meinhold et al. (2008) rutile source discriminating diagram

The maximum Nb values 4814 and 3454 ppm were obtained in sample NSG2 and NSG13 which also content the highest Ta values (Table 2). With $\mathrm{Nb} / \mathrm{Ta}>1 \mathrm{ppm}$, the Nsanaragati rutile megaclasts fall within the range of Niobium rutile of Cerny et al. (1964). The high $\mathrm{Nb}$ values fall within those range (0.3 to $0.5 \mathrm{wt} . \%)$ of Rudnick et al. (2000) metasomatic rutile. For Rosendaal et al. (2009), rutile with high $\mathrm{Nb}$ and $\mathrm{Ta}$, and displaying $\mathrm{W}, \mathrm{Nb}, \mathrm{Sc}$, and Sn correlations, often crystallized in felsic environment. If based on this interpretation, felsic crystallization will be suggested for greyish rutile grains with high $\mathrm{Nb}$ and $\mathrm{Ta}$ contents, and showing $\mathrm{Nb}, \mathrm{Sc}$ and $\mathrm{Sn}$ correlations. $\mathrm{The} \mathrm{Nb} / \mathrm{Ta}$ ratios for some of the rutile match the recorded values for Rudnick et al. (2000) and crustal and eclogitic rutile.

\subsection{Petrogenesis}

$\mathrm{Al}$ and $\mathrm{Mg}$ behavior in rutile have been used to discriminate those from crustal and mantle origin (Smyth et al., 2008). The plotted $\mathrm{Al}_{2} \mathrm{O}_{3}$ and $\mathrm{MgO}$ abundances in Smyth et al. (2008) rutile sources discrimination diagram (Figure 7) shows that most of the rutile are affiliated to a crustal crystallization environment, with just a single grain been related to mantle crystallization. Although, $\mathrm{Fe}_{2} \mathrm{O}_{3}$ contents were not quantified, $\mathrm{FeO}$ enrichment in the Nsanaragati rutile grains may shows rutile preference in $\mathrm{Fe}^{2+}$ than $\mathrm{Fe}^{3+}$ as suggested by Cerny et al. (2007). 


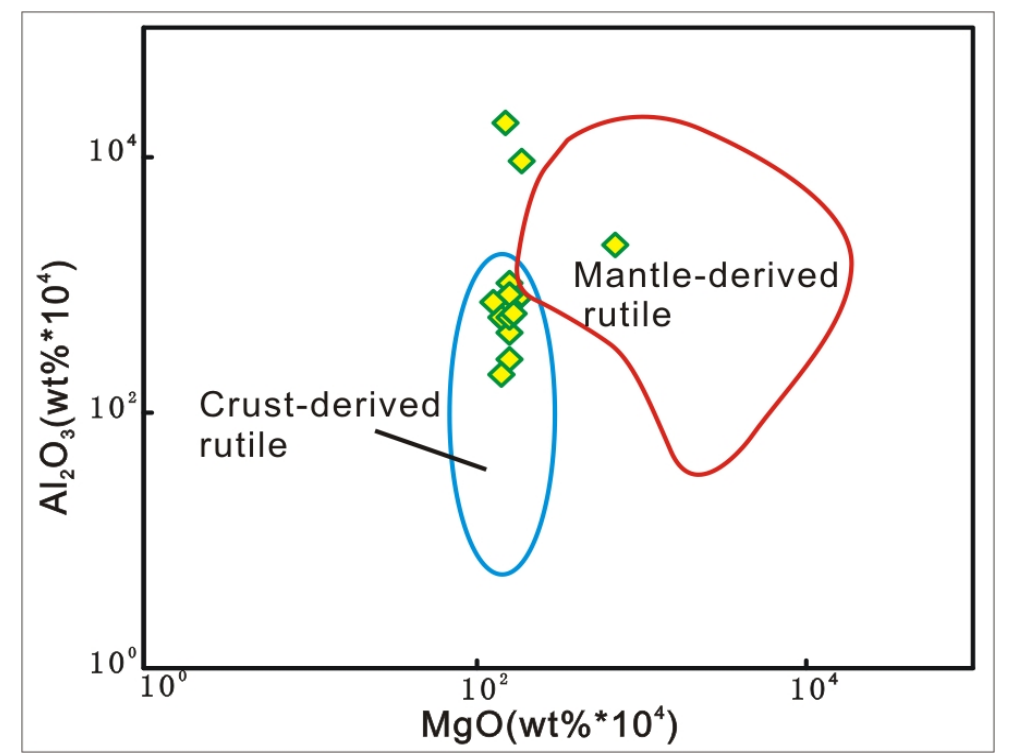

Figure 7. Nsanaragati rutile $\mathrm{Al}_{2} \mathrm{O}_{3}$ and $\mathrm{MgO}$ abundance plotted in Smythe et al. (2008) rutile source discriminating diagram

$\mathrm{Cr}, \mathrm{Nb}, \mathrm{Zr}$, Ta and Hf are known as good source constrainers for rutile (Zack et al., 2004a, 2004b; Luvizotto and Zack, 2009; Meinhold et al., 2008; Meinhold, 2010). For example the $\mathrm{Nb}$ ( 964-4817 ppm) and Cr (495-756 ppm) contents (Table 2 and 3) are within the range values of metapelitic rutile ( Triebold et al., 2007; Meinhold et al., 2008) consistent with their main crystallization environment. However some of the studied rutile grains display moderate $\mathrm{Cr}$ and $\mathrm{V}$ correlation that may indicate mafic igneous contributions (Rosendaal et al., 2009) whereas the two highest $\mathrm{Nb}$ contents 3454 and 4814 in ppm are within the range of metasomatic rutile (3000-5000 ppm) of Rudnick et al. (2000). Silica fluid contamination from surrounding rocks, can be suggested here to explain the high Si content in some of rutile grains ( Zack et al., 2004a; 2004b; Luvizotto and Zack, 2009). The fact that the studied rutile grains have high $\mathrm{Nb}$ and Ta contents and display $\mathrm{Nb}, \mathrm{Sc}$ and $\mathrm{Sn}$ correlations, is mostly consistent with their crystallization in felsic environment, following the result obtained by Rosendaal et al. (2009) for other studied rutile crystals.

\subsection{Zr Thermometer}

$\mathrm{Zr}$ abundance in rutile has been used for thermometric measuring and source constraining (Zack et al., 2004a, 2004b; Luvizotto \& Zack, 2009; Meinhold, 2010). Zr versus T plot diagram (Figure 8) confirms the existing correlation between these two parameters in rutile. The calculated crystallization temperature using Zack et al. (2004b) equation ranges from 470 to $675^{\circ} \mathrm{C}$ (Table 2). They show that rutile with temperature ranging from 470 to $550{ }^{\circ} \mathrm{C}$ were crystallized in greenschist and bleuschists while those whose temperature ranges from 550 to 675 ${ }^{\circ} \mathrm{C}$ has amphibolitic to eclogitic affinities as presented in Morton \& Chenery (2009) and Meinhold (2010) rutile thermometric table (Table 5). 


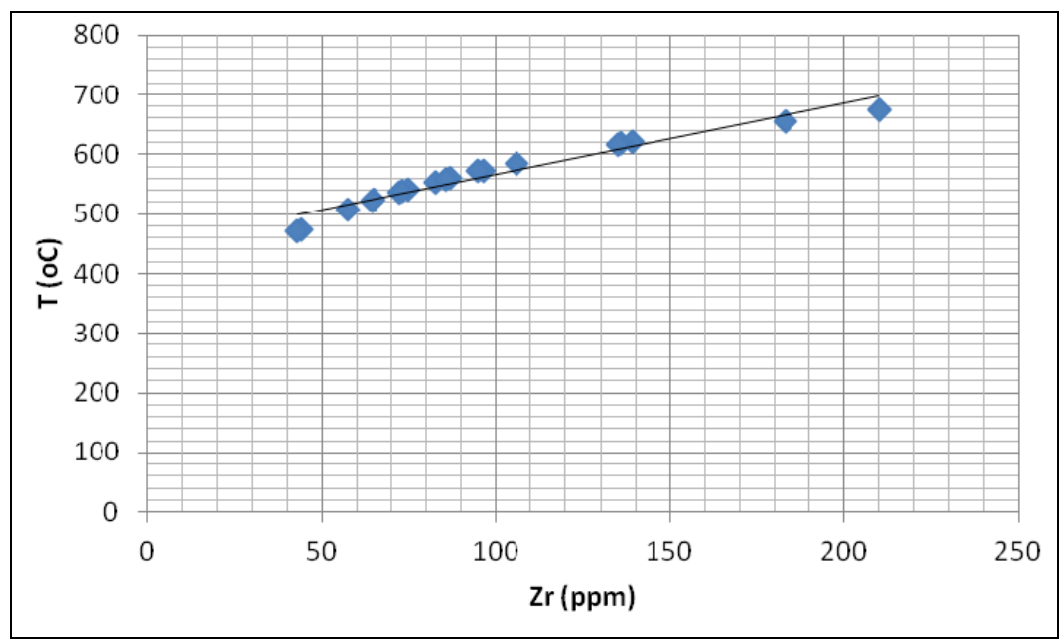

Figure 8. Temperature versus $\mathrm{Zr}$ correlation plot diagram for the Nsanaragati greyish-black rutile megaclasts

Table 5. Potential source rocks for the Nsanaragati rutile megaclasts based on Morton and Chenery (2009) and Meinhold (2010) rutile crystallization range temperature

\begin{tabular}{lccc}
\hline \multicolumn{1}{c}{ Sample } & Temperature range & Potential source rocks \\
\hline NSG04, NSG06, NSGO9, NSG11, NSG12, NSG15, & \multirow{2}{*}{470 to 550 } & Greenschist-blueschist \\
NSG17, NSG18, & & \\
NSG01, NSG02, NSG03, NSG05, NSG07, NSG08, NSG10, & 550 to 675 & Amphibolite-Eclogite \\
NSG13, NSG14, NSG16, NSG19 & &
\end{tabular}

\section{Conclusion}

The Nsanaragati greyish black rutile megaclasts are eroded product from local sedimentation and long distance hydrodynamic transportation that were probably sorted from coarse to very coarse grained rocks. They are chemically enriched rutile grains mostly grown in metapelitic source rocks.

\section{Acknowledgements}

Special thanks for UNESCO and Chinese Government for the funding of the laboratory analyses. Thanks are also due to the personals at the Key state laboratory in Geological Processes and Mineral Resources, China University of Geosciences, for their assistance during the analytical phases of the rutile. We will also thank the reviewers for their contribution in the improvement of the manuscript.

\section{References}

Benkhelil, J. (1989). The origin and evolution of the Cretaceous Benue Trough (Nigeria). Jour. African. Earth. Sciences, 8, 251-282. http://dx.doi.org/10.1016/S0899-5362(89)80028-4

Berthois, L. (1975). Etude sédimentologique des roches meubles (techniques et méthodes), Doin éditeur, paris, $277 \mathrm{p}$.

Birch, W. D., Barrons, L. M., Magee, C., \& Sutherland, F. L. (2007). Gold- and diamond-bearing White Hills gravel, St Arnaud district, Victoria: age and provenance based on U-Pb dating of zircon and rutile. Australian Journal Earth Sciences, 54, 609-628. http://dx.doi.org/10.1080/08120090701188996

Bromiley, G. D., \& Hilairet, N. (2005). Hydrogen and minor element incorporation in synthetic rutile. Mineral. Mag. 69, 345-358. http://dx.doi.org/10.1180/0026461056930256

Cailleux, L. (1946). Distinction des sables marins et fluviatiles. Bull .Soc. Geo. France, 125-138.

Černý, P., Čech, F., \& Povondra, P. (1964). Review of ilmenorutile-struvertite minerals. Neues Jahrbuch für Mineralogie Abhandlungen, 101, 142-172.

Černý. P., Novak, M., Chapman. R., \& Ferreira, K. (2007). Subsolidus behaviour of niobian rutile from the Pisek region, Czech Republic. Geosc., 25, 143-159. 
Cherniak, J. D., Manchester, J., \& Watson, B. E. (2007). Zr and Hf diffusion in rutile. Earth and Planetary Sciences Letters, 261, 267-279. http://dx.doi.org/10.1016/j.eps1.2007.06.027

Clack, R, J., \& Williams-Jones, E. A. (2004). Rutile as a potential indicator mineral for metamorphosed metallic ore deposits. Rapport Final de DIVEX, Sous-projet SC2, Montréal, Canada. 17 pp.

Dill, H. G., Melcher, F., Fu" B1, M., \& Weber, B. (2007). The origin of rutile-ilmenite aggregates ("nigrine") in alluvial-fluvial placers of the Hagendorf pegmatite province, NE Bavaria, Germany. Jour. Mineral. Petrol, 89, 133-158. http://dx.doi.org/10.1007/s00710-006-0140-x

Dostal, J., Kontak, J. D., \& Chatterjee, A. K. (2009). Trace element geochemistry of scheelite and rutile from metaturbidite-hosted quartz vein gold deposits, Meguma Terrane, Nova Scotia, Canada: genetic implications. Jour. Mineral. Petrol, 97, 95-109. http://dx.doi.org/10.1007/s00710-009-0067-0

Dumort, J. C. (1968). Carte géologique de reconnaissance du Cameroun à l'échelle 1/500000 feuille Douala-Ouest, avec notice explicative. Imprimerie Nationale, Yaoundé Cameroun, 69p.

Duplaix, S. (1958). Détermination microscopique des minéraux des sables. $2^{\mathrm{e}}$ édition révisée, Paris, 571p.

Eyong, J. T. (2003). Lithostratigraphy of the Mamfe cretaceous basin. South West province of Cameroon-West Africa. Ph.D. thesis, University of Leeds, 256p.

Ferry, J. M., \& Watson, E. B. (2007). New thermodynamic model and revised calculations for the Ti-in zircon and Zr-in-rutile thermometers. Contrib. Mineral. Petrol, 154, 429-437. http://dx.doi.org/10.1007/s00410-007-0201-0

Garda, M. G., Beljavskis, P., D’Argostino, Z. L., \& Wiedenbeck, M. (2010). Tourmaline and rutile as indicators of a magmatic -hydrothermal origin for tourmalinite layers in Sao Jose do Barreiro Area, NE Ribeira belt, Southern Brazil. Geologia USP, 10, 97-117.

Green, H. T. (1995). Significance of $\mathrm{Nb} / \mathrm{Ta}$ as an indicator of geochemical process in the crust- mantle systern. Chem. Geol, 120, 347-359. http://dx.doi.org/10.1016/0009-2541(94)00145-X

Hu, Z. C., Gao, S., Liu, Y. S., Hu, S. H., Chen, H. H., \& Yuan, H. L. (2008). Signal enhancement in laser ablation ICP-MS by addition of nitrogen in the central channel gas. Jour. Analy. Atomic. Spectrometry, 23, 1093-1101. http://dx.doi.org/10.1039/b804760j

James, D. D. (1851). Manual of Mineralogy: Including Observations on Mines, Rocks, Reductions of ores, and the Application of the Sciences of the Arts, With 260 illustration (3rd ed.). Published by Durrie and Peck. Philadelphia: Horace C. Peck, 432p.

Kanouo, S. N. (2008). Etude géologique des indices de minéralisation de saphir dans la partie Sud du bassin sédimentaire de Mamfe. Mémoire de D.E.A, Université de Yaoundé I, 91 .

Kanouo, S. N., Khin Zaw, Yongue, F. R., Sutherland, L. F., Meffre, S., Njonfang, E.,...Tchouatcha, S. M. (2012). $\mathrm{U}-\mathrm{Pb}$ zircon age constraining the source and provenance of gem-bearing Late Cenozoic detrital deposits, Mamfe Basin, SW Cameroon. Journal of Resources Geology (in press).

Klemme, S., Prowatke, S., Hametner, K., \& Gunther, D. (2005). Partitioning of trace elements between rutile and silicate melts: implications for subduction zones. Geochim. Cosmochim Acta, 69, 2361-2371. http://dx.doi.org/10.1016/j.gca.2004.11.015

Laplaine, L., \& Soba, D. (1967). Rapport du service géologique pour les années 1965-1966-1967, prospection de saphirs dans le bassin crétacé de Mamfé. Archives BRGM /DMG/ MINMEE.

Le Fur, Y. (1964-1965). Rapport spécial sur les recherches de corindons gemmes. Mission socle-crétacé, Mamfé YAO 666A8. Archives BRGM. DMG / MINEE.

Liu, Y. S., Hu, Z. C., Gao, S., Günther, D., Xu, J., Gao, C. G.,...Chen, H. H. (2008). In situ analysis of major and trace elements of anhydrous minerals by LA-ICP-MS without applying an internal standard. Chemical Geology, 257, 34-43. http://dx.doi.org/10.1016/j.chemgeo.2008.08.004

Luvizotto, L. G., \& Zack, T. (2009). Nb and $\mathrm{Zr}$ behavior in rutile during high-grade metamorphism and retrogression: An example from the Ivrea-Verbano Zone. Chemical Geology, 261, 302-317. http://dx.doi.org/10.1016/j.chemgeo.2008.07.023

Mange, A. M., \& Maurer, W. F. H. (1992). Heavy minerals in colour. Chapman and Hall, 147p. http://dx.doi.org/10.1007/978-94-011-2308-2

McDonough, F. W., \& Sun, S. S. (2005). The composition of the Earth. Chem. Geol, 120, 223-253. http://dx.doi.org/10.1016/0009-2541(94)00140-4 
Meinhlod, G., Andres, B., Kostopoulos, D., \& Reischmann, T. (2008). Rutile chemistry and thermometry as provenance indicator: An example from Chios Island, Greece. Sed Geol, 203, 98-111. http://dx.doi.org/10.1016/j.sedgeo.2007.11.004

Meinhold, G. (2010). Rutile and its application in earth sciences. Earth. Sc. Reviews, 102, 1-28. http://dx.doi.org/10.1016/j.earscirev.2010.06.001

Miller, C., Zanetti, A., Thoni, M., \& Konzett, J. (2007). Eclogitisation of gabbroic rocks: Redistrubution of trace elements and $\mathrm{Zr}$ in thermometry in an Eo-Alpine subduction zone (Eastern Alps). Chem Geol, 239, 96-123. http://dx.doi.org/10.1016/j.chemgeo.2007.01.001

Morton, A., \& Chenery, S. (2009). Detrital rutile geochemistry and thermometry as guides to provenance of Jurassic -Paleocene sandstones of the Norvegian Sea. Sed. Research, 79, 540-553.

Myron, G. B. (2003). Igneous and metamorphic petrology (2nd ed.). Blackwell Sicences Ltd, 16-49.

Parfenoff, A., Pomerol, C., \& Tourenq, J. (1970). Les minéraux en grains. Méthodes d'étude et détermination. Masson et Cie. Edit. Paris, 571p.

Pellant, C. (1994). Roches et Minéraux : le guide visuel de plus de 500 spécimens des roches et minéraux à travers le monde. Eye witness Handbook- rocks and minerals, 256p.

Roux, P. J., \& Rojax, M. E. (2007). Sediment transport patterns determined from grain size parameters: Overview and state of the art. Jour. Sed. Geol, 202, 473-488. http://dx.doi.org/10.1016/j.sedgeo.2007.03.014

Rozendaal, A., Philander, C., \& Carelse, C. (2009). Characteristics, recovery and provenance of rutile from the Namakawa sand heavy mineral deposit, South Africa. The $7^{\text {th }}$ International Heavy Mineral Conference $<$ what next $>$, the South African institute of Mining and Metallurgy.

Rudnick, L. R., Barth, M., Horn, I., \& McDonough, F. W. (2000). Rutile-Bearing Refractory Eclogites: Missing Link Between Continents and Depleted Mantle. Science, 287, 278-281. http://dx.doi.org/10.1126/science.287.5451.278

Scott, K. M. (2005). Rutile geochemistry as a guide to porphyry $\mathrm{Cu}-\mathrm{Au}$ mineralization, Northparkes, New South Wales, Australia. Geochemistry: Exploration, Environment Analysis, 5, 247-253. http://dx.doi.org/10.1144/1467-7873/03-055

Selley, C. R. (1982). An introduction to sedimentology. Academic Press, 417p.

Smythe, D. J., Schulze, D. J., \& Brenan, J. M. (2008). Rutile as a kimberlite indicator mineral: minor and trace element geochemistry. $9^{\text {th }}$ International Kimberlite Conference Extended Abstract, No 9IKC-A-00193.

Stendal, H., Toteu, F. S., Frei, R., Penaye, J., Njel, O. U., Bassahak, J.,...Hell, V. J. (2006). Derivation of detrital rutile in Yaoundé region from Cameroon the Neoproterozoic Pan-Africain belt in southern Cameroon (Central Africa). Jour. African Earth Sciences, 44, 443-458. http://dx.doi.org/10.1016/j.jafrearsci.2005.11.012

Triebold, S., Eynatten, V. H., Luvizotto, L. G., \& Zack, T. (2007). Deducing source rock lithology from detrital rutile geochemistry: Example from the Erzgebirge, Germany. Chem. Geol, 244, 421-436. http://dx.doi.org/10.1016/j.chemgeo.2007.06.033

Wentworth, K. C. (1922). A scale of grade and class terms for clastic sediments. J. Geology, 30, 377-392. http://dx.doi.org/10.1086/622910

Wilson, D. (1928). Notes on the geology of the Mamfe Division, Cameroon SW province. Occasional papers. Geology survey Nigeria ${ }^{\circ} 6$.

Xiong, L. X., Adam, J., \& Green, H. T. (2005). Rutile stability and rutile/melt HFSE partitioning during partial melting of hydrous basalt: Implications for TTG genesis. Chem. Geol, 218, 339-359. http://dx.doi.org/10.1016/j.chemgeo.2005.01.014

Zack, T., Eynatten, V. H., \& Kronz, A. (2004b). Rutile geochemistry and its potential use in quantitative provenance studies. Journal of Sedimentary Geology, 171, 37-58. http://dx.doi.org/10.1016/j.sedgeo.2004.05.009

Zack, T., Kronz, A., Foley, S. F., \& Rivers, T. (2002). Trace element abundances in rutiles from eclogites and associated garnet mica schists. Chemical Geology, 184, 97-122. http://dx.doi.org/10.1016/S0009-2541(01)00357-6

Zack, T., Moraes, R., \& Kronz, A. (2004a). Temperature dependence of $\mathrm{Zr}$ in rutile: empirical calibration of a rutile thermometer. Contrib. Mineral. Petrol, 148, 471-488. http://dx.doi.org/10.1007/s00410-004-0617-8 